\title{
Lipophilic Probe Behavior in Microemulsions Evaluated by Fluorescence Correlation Spectroscopy
}

Johtaro YAMAMOTO,* Yoshio SUZUKI,* Yoshikatsu OGAWA,* Tomoyuki KAMATA,* Hinako HASHIMOTO,*** Masashi KUNITAKE,** and Dai KATO*广

* Health and Medical Research Institute, National Institute of Advanced Industrial Science and Technology (AIST), 1-1-1 Higashi, Tsukuba, 305-8566, Japan.

** Institute of Industrial Nanomaterials, Kumamoto University, 2-39-1 Kurokami, Kumamoto 860-8555, Japan.

$\dagger$ To whom correspondence should be addressed.

E-mail: dai.kato@aist.go.jp 


\begin{abstract}
We evaluated the dispersion and diffusion of fluorescent-labeled lipophilic vitamin $\mathrm{E}(\mathrm{VE})$ in microemulsions (MEs) including water-in-oil (W/O) type ME, oil-in-water $(\mathrm{O} / \mathrm{W})$ type $\mathrm{ME}$ and bicontinuous $\mathrm{ME}(\mathrm{BME})$, by using fluorescence correlation spectroscopy (FCS). We prepared a fluorescent ATTO 488 or BODIPY group labeled VE (VE-ATTO or VE-BODIPY). VE-ATTO possesses lipophilic and hydrophilic parts, while VE-BODIPY consists solely of the lipophilic part. The VE-ATTO dissolved in heptane solution as an oil phase, appeared hot pink in color due to the solvatochromism effect under room light and almost no fluorescent signal, which was unlike the VE-ATTO dissolved in ME solutions and all the VE-BODIPY solutions (typical fluorescent green color). The FCS measurement rapidly proved that VE-BODIPY diffuses faster than VE-ATTO. This is presumably because the "surfactant-like" VE-ATTO is localized and trapped at the micro-water/micro-oil interface of the MEs, while the VE-BODIPY exists in the ME phase and macro-oil phase with good dispersion. These results demonstrate that FCS is a powerful tool for the rapid evaluation of the lipophilic probe behavior in heterogeneous ME solutions.
\end{abstract}




\section{Introduction}

The homogeneous dispersion of chemicals is a significant requirement in various application fields such as pharmaceuticals and cosmetics. ${ }^{1-3}$ In particular, the solubility of target substances in colloidal solutions such as microemulsions (MEs) is very important because MEs consisting of water, oil, and surfactant (frequently co-surfactant) are dispersed solutions that solubilize both lipophilic and hydrophilic substances. ${ }^{4}$ Homogeneous dispersions for MEs can generally be evaluated by electron microscopy, scattering techniques (e.g., light, X-rays and neutrons), viscosity, fluorescence correlation spectroscopy (FCS) and so on. ${ }^{1,5}$ On the other hand, the ME structure is mainly classified as an oil-in-water $(\mathrm{O} / \mathrm{W})$, water-in-oil $(\mathrm{O} / \mathrm{W})$ or bicontinuous structure, depending on the hydrophilic-lipophilic balance of the surfactant. Among them, a bicontinuous ME (BME) solution has unique properties that include not only the solubility of both hydrophilic and lipophilic substances simultaneously but also conductivity as a result of the bicontinuous structure. ${ }^{6,7}$ Therefore, BME is considered to be a suitable solution for use in performing electrochemical measurements. Indeed, Rusling et al. have reported on the electrochemistry in a BME with the result that electrochemical reactions with both hydrophilic and lipophilic redox substances have become possible with the same BME solution. ${ }^{6,8}$ One of our co-authors, Kunitake has also been studying electrochemistry in BME (BME-EC). ${ }^{4,},{ }^{10} \mathrm{He}$ investigated the properties of BME-EC by using various electrode materials and found BME structures at an oil/water/electrode ternary interface that can be easily changed depending on the property of the electrode surface. Indeed, Kuraya et al. reported the selective and individual determination of hydrophilic and lipophilic antioxidants in the same BME solution. ${ }^{11,12}$ Here, a hydrophobic fluorinated nanocarbon (F-nanocarbon) film electrode produced the oxidation current of lipophilic $\alpha$-tocopherol (vitamin E, VE), but no 
oxidation peak for hydrophilic L-ascorbic acid (vitamin C, VC). In contrast, hydrophilic indium tin oxide electrode exhibited the oxidation response of VC but not of VE. From these results, we consider that hydrophilic and lipophilic compounds diffuse through micro-water and the micro-oil phase in BME, respectively. Moreover, very recently, we realized lipophilic VE diffusion through BMEs by using both electrochemical and fluorescence correlation spectroscopy (FCS) measurements. ${ }^{13}$ The VE diffusion information obtained in the electrochemical and FCS experiments are related to a region near the interface between the electrode surface and the BME solution, and a bulk BME solution, respectively. We found that the VE diffusion accelerated as the micro-oil content of the BME increased due to the widening of the micro-oil phase pathway in the BME. To achieve an FCS evaluation of VE diffusion through BME requires the fluorescent labeling of VE because the fluorescent property of VE itself is very weak. Therefore, we used BODIPY labeled VE (VE-BODIPY). There are excellent fluorescent groups thanks to the great efforts of many researchers. We must focus on selecting suitable fluorescent groups for FCS evaluation in terms of wavelength, concentrations, and hydrophilicity/hydrophobicity. In particular, when lipophilic probes are used in heterogeneous ME, their hydrophilic/lipophilic balance is an important consideration in the modification of fluorescent groups to lipophilic substances. For example, given that a hydrophilic fluorescent group is labeled with a lipophilic target, the resulting probe will behave like a surfactant that induces aggregation formation such as micelles in aqueous solvents or reverse micelles in organic solvents including MEs.

In this study, we prepared two lipophilic probes with different chemical structures and evaluated the dispersion and diffusion of these probes in heterogeneous MEs by using FCS measurements. 


\section{Experimental}

\section{Reagents and chemicals}

2-butanol (2-BuOH), sodium dodecyl sulfate (SDS), heptane, and sodium nitrate $\left(\mathrm{NaNO}_{3}\right)$ were purchased from Fujifilm WAKO (Japan). VE was purchased from Nacalai Tesque (Japan). ATTO488 NHS-ester and BODIPYTM FL NHS Ester were purchased from ATTO-TEC GmbH (Germany) and ThermoFisher Scientific (USA), respectively. All the chemicals were analytical grade and were used as received. Ultrapure water was used in all the experiments.

\section{Preparation and observation of ME systems}

ME solutions were prepared in accordance with previous reports. ${ }^{11-13}$ A mother emulsion solution, comprising 1.0 $\mathrm{M} \mathrm{NaNO}_{3}$ aqueous solution (water phase, $5 \mathrm{~mL}$ ), SDS (surfactant, $0.45 \mathrm{~g}$ ), heptane (oil phase, $5 \mathrm{~mL}$ ), and 2-BuOH (cosurfactant, 2.1-3.9 $\mathrm{mL}$ ) was mixed and vortexed, and the solution was allowed to stand at room temperature for more than $2 \mathrm{~h}$. O/W type, W/O type, and BME were prepared by adjusting the volume of the 2-BuOH. We macroscopically observed a two or three-phase solution, consisting of macro-water, middle (BME), and macro-oil phases as seen by the naked eye although these ME solutions have high transparency. However, when taking a photograph under usual room lights (2600-2700 lumens), it was difficult to distinguish the appearance. Therefore, a very bright light (9000 lumens) was irradiated from the bottom of the test tube to emphasize the scattering of the ME phase (Tyndall effect) while the photographs were taken (Fig. 1(b). ${ }^{13}$ After that, the macro-water, the macro-oil, and the middle phases were gently extracted as the $\mathrm{O} / \mathrm{W}$ type ME, W/O type ME, and BME solution, respectively. These ME solutions were 
confirmed microscopically by double staining the water-soluble (anionic) calcein (2.0 $\mu \mathrm{M})$ (Dojindo, Japan) for the aqueous phase, and fat-soluble (nonionic) Nile red (2.0 $\mu \mathrm{M}$ ) (Wako) for the oil phase. The ME solutions were observed using an Olympus IX70 fluorescence microscope (an Objective lens: Olympus UPlanSApo 10x, N.A. 0.40) and a Hamamatsu Orca-ER CCD Camera. Calcein and Nile red were imaged using an Olympus filter set U-MGFPHQ (460-480 $\mathrm{nm}$ excitation filter, 495-540 $\mathrm{nm}$ bandpass emission filter) and an Olympus filter set U-MWG2 (510-550 nm excitation filter, $>590$ $\mathrm{nm}$ longpass emission filter), respectively. Digital images captured with a CCD camera were processed using Photoshop (Adobe Systems, USA).

\section{Synthesis of fluorescent labeled VE}

To assess the dispersion property of lipophilic probes in various solvents by FCS measurement, we synthesized two types of lipophilic VE probes, namely fluorescent groups (ATTO 488 and BODIPY) labeled VE (namely, VE-ATTO and VE-BODIPY, Fig. 2). The VE-ATTO was synthesized as shown in Supporting Information. The VE-BODIPY was synthesized in accordance with our previous report. ${ }^{13}$ The synthesized probes were long-term stable without decomposition under light protection storage at $-20{ }^{\circ} \mathrm{C}$ in powder form. These probes were stored as a powder at $-20{ }^{\circ} \mathrm{C}$ under light protection until use. The probe solution was prepared during each experiment to avoid the effect of changes in probe concentration caused by volatilization of organic solvents.

\section{Preparation and observation of VE probe solutions}

The synthesized VE probes were dissolved in heptane, and the extracted $\mathrm{W} / \mathrm{O}, \mathrm{O} / \mathrm{W}$, and BME solutions. The final concentrations of the VE probes were $100 \mu \mathrm{M}$ (Fig. 3). In 
contrast, these probes were dissolved in a mixture of heptane and $1 \mathrm{M} \mathrm{NaNO} \mathrm{Na}_{3}$ aqueous solution $(\mathrm{O}+\mathrm{W}), \mathrm{W} / \mathrm{O}, \mathrm{O} / \mathrm{W}$, and BME-based solutions (not in the extracted ME parts, Fig. 4). In this case, the final concentrations of the VE probes were $1 \mu \mathrm{M}$. The VE probe solutions were observed under room light and UV light.

\section{FCS measurement}

After preparing the ME solutions, we added the VE-BODIPY or VE-ATTO with a final concentration of $100 \mathrm{nM}$ with a heptane volume of $5 \mathrm{~mL}$. This probe concentration is a typical concentration suitable for performing FCS measurements. ${ }^{14}$ The ME solutions with these probes $(1.0 \mathrm{~mL})$ were placed on a glass bottom dish (dish diameter $35 \mathrm{~mm}$, glass diameter $14 \mathrm{~mm}$, glass thickness 0.16-0.19 mm, Matsunami Glass, Japan) and the FCS measurement was conducted as follows. A polarization-dependent fluorescence correlation spectroscopy (Pol-FCS) system $^{15}$ was used to perform an FCS measurement on these probes in each solution. The detail of the Pol-FCS system has been reported previously. ${ }^{13}$ The focal point of the objective lens was placed in each solution sample on the glass bottom dish. The excitation laser power was $303 \mu \mathrm{W}$ at the focal plane of the objective lens. The 10-s measurement was repeated 12 times to obtain one averaged cross-correlation function $(\mathrm{CCF})$. A non-linear least squares analysis was performed to analyze the average CCF using the two component model equation $(G(\tau)$, Eq. 1) defined as follows ${ }^{16}$ :

(Eq 1)

where $\tau, N$, and $F_{1}$ denote the delay time, the average number of molecules inside the focal volume, and the fraction of the first decaying component of $\mathrm{CCF}$, respectively. 
$\tau_{1}$ is the characteristic decay time of the first decaying component of the CCF called the diffusion time in FCS. The diffusion time is inversely proportional to the diffusion coefficient. A longer diffusion time indicates a slower diffusion of target molecules. $\tau_{2}$ is the characteristic decay time, which is slower than the first decaying component characterized by $\tau_{2}$. In this study, this slower component rarely appeared. The slower component was likely due to the movement of relatively large water droplets near the surface of the cover slip. The number of target molecules was no longer appropriate because of the existence of non-diffusion component. Therefore, we do not mention the number of target molecules or the photon counts per molecules (CPM) in this study. $s$ is the ratio between the axial radius and the lateral radius of the focal volume called the structure parameter.

\section{Results and Discussion}

Preparation and observation of ME solutions.

Fig. 1 (a) shows a typical phase change with increasing 2-BuOH addition. First, a two phase solution consisting of $\mathrm{O} / \mathrm{W}$ type $\mathrm{ME}$ and macro-oil phase $(\mathrm{O} / \mathrm{W}+\mathrm{O})$ is formed. It is then changed to a three phase solution consisting of BME, macro-oil, and macro-water phases $(\mathrm{O}+\mathrm{BME}+\mathrm{W})$. Finally, the solution is changed to a two phase solution consisting of W/O type $\mathrm{ME}$ and the macro-water phase $(\mathrm{W} / \mathrm{O}+\mathrm{W})$ as shown in Fig. 1 (b and c). Note that the scale of the BME illustration in Fig. 1(c) differs from the $\mathrm{W} / \mathrm{O}$ and $\mathrm{O} / \mathrm{W}$ illustrations or the microscopic images in Fig. 1(d); it is illustrated more microscopically. We also observed both $\mathrm{O} / \mathrm{W}$ and W/O type MEs by using fluorescent imaging with double staining (Fig. 1 (d)). With BME, no clear structure could be observed as shown in Fig. 1 (d). This is because the size of the BME structure is below 
the diffraction limit of light, although Nazar and co-workers successfully observed the BME structure (typically around $100 \mathrm{~nm}$ ) with an optical microscope. ${ }^{17,18}$ Nevertheless, the fluorescent images exhibited fluorescent signals based on both calcein and Nile red. These results indicate that both phases coexisted in this BME solution.

Observation of lipophilic probes in ME solutions.

We prepared two types of lipophilic probes, VE-ATTO and VE-BODIPY, as shown in Fig. 2. It is well known that both ATTO 488 and BODIPY exhibit strong fluorescent signals around a $480 \mathrm{~nm}$ excitation wavelength. The difference between them is the hydrophilic/lipophilic property of these structures. Since ATTO 488 has two sulfonate groups and two amino groups (totally neutral), the VE-ATTO molecule has both hydrophilic and lipophilic moieties. In contrast, BODIPY has solely a lipophilic group (nonionic) and is therefore lipophilic as an entire VE-BODIPY molecule. Molecular lipophilicity was also estimated from Clog $P$ values, which were obtained from a calculation with ChemDraw20.1.1. The Clog $P$ values are 8.01 for VE-ATTO and 14.94 for VE-BODIPY. This large difference was attributed to the Clog $P$ values of each fluorescent part for the probes, namely -3.69 for ATTO 488 and 3.45 for BODIPY, whereas it was 12.05 for VE.

We confirmed the solubility of these probes in heptane (oil phase) and three types of ME solutions. Fig. 3 shows photographs of each probe dissolved in perspective solutions. VE-ATTO dissolved in heptane as an oil phase, was hot pink in color under room light and an almost non-fluorescent green color under UV light. On the other hand, VE-ATTO dissolved in $\mathrm{O} / \mathrm{W}, \mathrm{W} / \mathrm{O}$, and BME solutions was a typical fluorescent green color, which was unlike the VE-ATTO/heptane sample. This is probably because 
VE-ATTO with both hydrophilic and lipophilic parts behaves as a "surfactant" and forms reverse micelles in heptane, inducing the solution to change color due to the solvatochromism effect, ${ }^{19}$ and lack of fluorescent signals. With VE-BODIPY, all the samples were fluorescent green irrespective of the solutions we used. These results allow us to deduce that the dispersion state of each probe is quite different despite the fact that all the probe solutions exhibited strong fluorescence except for the VE-ATTO/heptane (Fig. 3).

We then confirmed the dispersion and partition of these probes in these ME-based solutions (not in the extracted ME parts), as shown in Fig. 4. We observed that VE-BODIPY exists in ME phases and macro-oil phases. In contrast, VE-ATTO was observed only in ME phases, not in the macro-oil phase. Note that although VE-ATTO is not detected by fluorescence in the macro-oil phase, it is still present. From these results, the dispersion state of these probes under each condition is presumed to be as depicted in Fig. 4. That is, the VE-ATTO is localized and trapped at the micro-water/micro-oil interface, while the VE-BODIPY exists in ME phases and macro-oil phases. These differences may affect probe diffusion. To evaluate this, we conducted FCS measurements as described in a next section.

\section{FCS measurements.}

We evaluated the diffusion property of these lipophilic probes in various solutions by using FCS measurements. FCS measurement is one of the most appropriate techniques for estimating the diffusion of analyte in MEs because it provides various important pieces of information such as the number of target molecules and the photon count per molecule and their diffusion times. Indeed, some researchers have 
demonstrated that FCS can also be used for ME characterization as regards its diffusion, size, size distribution, and polydispersity. ${ }^{20-24} \mathrm{Pal}$ and co-workers successfully used an FCS system to measure the properties of MEs. ${ }^{20,}{ }^{22}$ However, there have been few reports concerning BME estimation using an FCS system. This would presumably be because a BME does not have a droplet shape. Neubauer and co-workers were the first to use FCS measurement to investigate the diffusion of an enhanced green fluorescent protein $(\mathrm{GFP}+)$ in a BME (water/sugar surfactant+1-pentanol/cyclohexane). ${ }^{25}$ They reported that the GFP+ diffusion slowed as the water domain size in the BME decreased. Recently, we also reported the VE diffusion through BME by using electrochemical and FCS methods with a VE-BODIPY probe. ${ }^{13}$

Fig. 5 shows typical CCFs of FCS measurements for VE-ATTO and VE-BODIPY dissolved in heptane (oil phase) and three types of ME solutions. It is noteworthy that the FCS results provided significantly different responses although the fluorescent characteristics obtained in Fig. 3 were almost the same. There were two decaying components in the normalized CCFs of VE-ATTO (Fig. 5(a)), a fast-decaying component in the $10^{-4}-10^{-2} \mathrm{~s}$ time range and a slow-decaying component in the $10^{-1}-10^{1}$ $\mathrm{s}$ time range. Similarly, the fast component of VE-BODIPY appeared in the $10^{-5}-10^{-2} \mathrm{~s}$ time range (Fig. 5(b)). The characteristic decay time of each component is called the diffusion time in FCS. The diffusion time of the fast component is fitted as $\tau_{1}$, and the slow component was fitted as $\tau_{2}$ in a non-linear least squares analysis with Eq. (1). The $\tau_{2}$ value probably corresponds with the movement of relatively large droplets as shown in Fig. 1 (c), which was not the components that we focused on. Furthermore, the number of molecules obtained with a fitting analysis using Eq. (1) is not the correct value if there are multiple species with different brightnesses. ${ }^{26}$ We therefore focused only on the fast component $\tau_{1}$ in the following. From a fitting analysis of the CCFs, we 
evaluated the diffusion times of each lipophilic probe under various conditions as shown in Fig. 6. In the fitting analysis, the structure parameter $s$ was fixed at 9.37 based on the fitting result for VE-BODIPY in heptane solution.

In the VE-ATTO samples, the diffusion time order became BME $(276 \mu$ s $)<\mathrm{W} / \mathrm{O}$ $(545 \mu \mathrm{s})<\mathrm{O} / \mathrm{W}(577 \mu \mathrm{s})$. This indicates that the VE-ATTO probe was trapped in the $\mathrm{W} / \mathrm{O}$ and $\mathrm{O} / \mathrm{W}$ droplets, thereby slowing the diffusion time. We observed no fluorescent signals with the VE-ATTO/heptane sample, which was coincident with the result in Fig. 3(a). With the VE-BODIPY samples, the diffusion time order was heptane $(31.9 \mu \mathrm{s})<$ $\mathrm{W} / \mathrm{O}(83.7 \mu \mathrm{s})<\mathrm{BME}(152 \mu \mathrm{s})<\mathrm{O} / \mathrm{W}(405 \mu \mathrm{s})$, and this is the reverse of the BME and W/O order from the results of the VE-ATTO samples. This was presumably because the diffusion was faster depending on the volumes of the continuous oil phase in the solutions. In addition, very interestingly, when we compared the diffusion times of the two probes in BME solution, the diffusion of the VE-ATTO probe was slower. This suggests that VE-ATTO is trapped or moved on the micro-water/micro-oil interface in the BME. In other ME solutions, VE-ATTO is localized in the micro-water/micro-oil interface in the MEs. Moreover, SDS is also localized at the micro-water/micro-oil interface. The SDS concentration is $156 \mathrm{mM}$, while the VE-ATTO concentration is 100 $\mathrm{nM}$ in these MEs. Although they differ greatly, they both have anionic sulfonates, which suggest that attractive stiffening occurs as a result of sharing the counterion $\mathrm{Na}^{+}$from 1 $\mathrm{M} \mathrm{NaNO}_{3}$ as the water phase. This may be reflected in the suppression of the VE-ATTO diffusion in all the MEs. On the other hand, the VE moiety alone may be incorporated in the SDS molecules by a hydrophobic effect, but in fact, the VE-BODIPY diffused relatively rapidly within the micro-oil phase of BME. This also supports the possibility of the "counterion-mediated stiffness of a micro-water/micro-oil interface" when using a VE-ATTO probe. This fact indicates that the VE-ATTO probe would be useful for the 
observation of the local structure of a micro-water/micro-oil interface if we use a super-resolution microscopy technique such as stimulated emission depletion (STED) microscopy. These results clearly demonstrate that dispersion and diffusion of the probe molecule is very important in terms of designing the hydrophilic/lipophilic property as an entire probe molecule, and the FCS allows the rapid evaluation of lipophilic probes dispersion and diffusion in heterogeneous ME solutions, which is difficult to achieve with conventional optical measurement including fluorometry.

\section{Conclusions}

We studied the dispersion and diffusion of lipophilic VE probes in heterogeneous ME solutions by using FCS. The FCS measurement rapidly revealed that the diffusion of VE-BODIPY was faster than that of VE-ATTO. This was presumably because the VE-ATTO was localized and trapped at the micro-water/micro-oil interface of MEs or formed reverse micelles in the macro-oil phase. On the other hand, VE-BODIPY exists in the ME phase and macro-oil phase with good dispersion. Therefore, VE-BODIPY is suitable as regards measuring its diffusion through the micro-oil phase in the MEs. ${ }^{13}$ This evaluation approach with FCS will be a powerful tool in a variety of fields, not only in designing lipophilic probes in this study, but also in easily and rapidly evaluating the dispersion state of chemicals in ME-related technologies such as emulsion polymerization and emulsion electrolysis.

\section{Acknowledgements}


We thank Azusa Hakuta for help with the BME experiments. This work was supported by a special strategic grant ("New-Generation Medical Treatment and Diagnosis Research Laboratory") from AIST (Japan).

\section{References}

1. Gradzielski, M.; Duvail, M.; de Molina, P. M.; Simon, M.; Talmon, Y.; Zemb, T., Using Microemulsions: Formulation Based on Knowledge of Their Mesostructure. Chem. Rev. 2021, 121 (10), 5671-5740.

2. Nastiti, C.; Ponto, T.; Abd, E.; Grice, J. E.; Benson, H. A. E.; Roberts, M. S., Topical Nano and Microemulsions for Skin Delivery'. Pharmaceutics 2017, 9 (4).

3. Callender, S. P.; Mathews, J. A.; Kobernyk, K.; Wettig, S. D., Microemulsion Utility in Pharmaceuticals: Implications for Multi-Drug Delivery. Int. J. Pharm. 2017, 526 (1-2), 425-442.

4. Kunitake, M.; Kuraya, E.; Kato, D.; Niwa, O.; Nishimi, T., Electrochemistry in Bicontinuous Microemulsions Based on Control of Dynamic Solution Structures on Electrode Surfaces. Curr. Opin. Colloid Interface Sci. 2016, 25, 13-26.

5. Acharya, D. P.; Hartley, P. G., Progress in Microemulsion Characterization. Curr. Opin. Colloid Interface Sci. 2012, 17 (5), 274-280.

6. Zhou, D. L.; Gao, J. X.; Rusling, J. F., Kinetic Control of Reactions of Electrogenerated Co(I) Macrocycles with Alkyl Bromides in a Bicontinuous Microemulsion. J. Am. Chem. Soc. 1995, $117(3), 1127-1134$.

7. Mackay, R. A.; Myers, S. A.; Bodalbhai, L.; Brajtertoth, A., Microemulsion Structure and Its Effect on Electrochemical Reactions. Anal. Chem. 1990, 62 (10), 1084-1090.

8. Iwunze, M. O.; Sucheta, A.; Rusling, J. F., Bicontinuous Microemulsions as Media for Electrochemical Studies. Anal. Chem. 1990, 62 (6), 644-649. 
9. $\quad$ Makita, Y.; Uemura, S.; Miyanari, N.; Kotegawa, T.; Kawano, S.; Nishimi, T.; Tominaga, M.; Nishiyama, K.; Kunitake, M., Electrochemical Investigation of Dynamic Solution Structures of Bicontinuous Microemulsion at Solid Interfaces. Chem. Lett. 2010, 39 (11), 1152-1154.

10. Yoshitake, S.; Ohira, A.; Tominaga, M.; Nishimi, T.; Sakata, M.; Hirayama, C.; Kunitake, M., Electrochemistry in Middle Phase Microemulsion Composed of Saline and Toluene with Sodium Dodecylsulfate and N-Butanol. Chem. Lett. 2002, 31 (3), 360-361.

11. Kuraya, E.; Nagatomo, S.; Sakata, K.; Kato, D.; Niwa, O.; Nishimi, T.; Kunitake, M., Simultaneous Electrochemical Analysis of Hydrophilic and Lipophilic Antioxidants in Bicontinuous Microemulsion. Anal. Chem. 2015, 87 (3), 1489-1493.

12. Kuraya, E.; Nagatomo, S.; Sakata, K.; Kato, D.; Niwa, O.; Nishimi, T.; Kunitake, M., Direct Analysis of Lipophilic Antioxidants of Olive Oils Using Bicontinuous Microemulsions. Anal. Chem. 2016, 88 (2), 1202-1209.

13. Kato, D.; Yamamoto, J.; Suzuki, Y.; Kamata, T.; Hashimoto, H.; Kunitake, M., Lipophilic Vitamin E Diffusion through Bicontinuous Microemulsions. Anal. Chem. 2021, 93 (42), $14231-14237$.

14. Sasaki, A.; Yamamoto, J.; Kinjo, M.; Noda, N., Absolute Quantification of RNA Molecules Using Fluorescence Correlation Spectroscopy with Certified Reference Materials. Anal. Chem. 2018, 90 (18), 10865-10871.

15. Yamamoto, J.; Matsui, A.; Gan, F.; Oura, M.; Ando, R.; Matsuda, T.; Gong, J. P.; Kinjo, M., Quantitative Evaluation of Macromolecular Crowding Environment Based on Translational and Rotational Diffusion Using Polarization Dependent Fluorescence Correlation Spectroscopy. Sci. Rep. 2021, $11(1), 10594$.

16. Fluorescence Correlation Spectroscopy. In Principles of Fluorescence Spectroscopy, Lakowicz, J. R., Ed. Springer US: Boston, MA, 2006; pp 797-840.

17. Nazar, M. F.; Mujeed, A.; Siddique, M. Y.; Zafar, M.; Saleem, M. A.; Khan, A. M.; 
Ashfaq, M.; Sumrra, S. H.; Zubair, M.; Zafar, M. N., Structural Dynamics of Tween-Based Microemulsions for Antimuscarinic Drug Mirabegron. Colloid Polym. Sci. 2020, 298 (3), 263-271.

18. Nazar, M. F.; Siddique, M. Y.; Saleem, M. A.; Zafar, M.; Nawaz, F.; Ashfaq, M.; Khan, A. M.; Rahman, H. M. A.; Tahir, M. B.; Lazim, A. M., Fourth-Generation Antibiotic Gatifloxacin Encapsulated by Microemulsions: Structural and Probing Dynamics. Langmuir 2018, 34 (36), 10603-10612.

19. Marini, A.; Munoz-Losa, A.; Biancardi, A.; Mennucci, B., What Is Solvatochromism? J. Phys. Chem. B 2010, 114 (51), 17128-17135.

20. Khan, M. F.; Singh, M. K.; Sen, S., Measuring Size, Size Distribution, and Polydispersity of Water-in-Oil Microemulsion Droplets Using Fluorescence Correlation Spectroscopy: Comparison to Dynamic Light Scattering. J. Phys. Chem. B 2016, 120 (5), 1008-1020.

21. Ghosh, S.; Mandal, A. K.; Das, A. K.; Mondal, T.; Bhattacharyya, K., Diffusion of Organic Dyes in a Niosome Immobilized on a Glass Surface Using Fluorescence Correlation Spectroscopy. Phys. Chem. Chem. Phys. 2012, 14 (27), 9749-9757.

22. Pal, N.; Verma, S. D.; Singh, M. K.; Sen, S., Fluorescence Correlation Spectroscopy: An Efficient Tool for Measuring Size, Size-Distribution and Polydispersity of Microemulsion Droplets in Solution. Anal. Chem. 2011, 83 (20), 7736-7744.

23. Burnett, G. R.; Rees, G. D.; Steytler, D. C.; Robinson, B. H., Fluorescence Correlation Spectroscopy of Water-in-Oil Microemulsions: An Application in Specific Characterisation of Droplets Containing Biomolecules. Colloid Surf. A 2004, 250 (1-3), 171-178.

24. Pyne, A.; Kuchlyan, J.; Maiti, C.; Dhara, D.; Sarkar, N., Cholesterol Based Surface Active Ionic Liquid That Can Form Microemulsions and Spontaneous Vesicles. Langmuir 2017, 33 (23), 5891-5899.

25. Neubauer, R.; Hohn, S.; Dulle, M.; Lapp, A.; Schulreich, C.; Hellweg, T., Protein Diffusion in a Bicontinuous Microemulsion: Inducing Sub-Diffusion by Tuning the Water Domain 
Size. Soft Matter 2017, 13 (10), 1998-2003.

26. Yamamoto, J.; Sasaki, A., Measurement of the Concentration and the Brightness for Samples Containing Multiple Molecules with Different Brightness Using Fluorescence Correlation Spectroscopy. Appl. Sci. 2021, 11 (13), 5840. 


\section{Figure Captions}

Fig. 1. (a) Typical phase changes of heptane/SDS+2-BuOH/1.0 $\mathrm{M} \mathrm{NaNO}$ aqueous solution microemulsions at room temperature, which are classified into three types of MEs, namely O/W type (blue), W/O type (green), and bicontinuous ME (BME) (red).

(b) Photographs of each ME. (c) Schematic illustrations and (d) fluorescent images of MEs by double staining. The photographs and fluorescent images of the samples were prepared under the conditions represented by each arrow. The 2-BuOH concentration at the points marked by arrows are $2.37,3.56,3.89 \mathrm{mM}$ for $\mathrm{O} / \mathrm{W}$ type $\mathrm{ME}, \mathrm{BME}, \mathrm{W} / \mathrm{O}$ type ME, respectively. The composition ratio of the micro-water phase and micro-oil phase in the BME (W/OBME) was 47/53.

Fig. 2. Chemical structures of synthesized lipophilic probes: (a) VE-ATTO and (b) VE-BODIPY.

Fig. 3. Photographs of (a) VE-ATTO and (b) VE-BODIPY dissolved in heptane, W/O, $\mathrm{O} / \mathrm{W}$, and BME solutions under room light and UV light. The probe concentration is 100 $\mu \mathrm{M}$.

Fig. 4. Photographs of (a) VE-ATTO and (b) VE-BODIPY dispersed in a mixture of heptane and $1 \mathrm{M} \mathrm{NaNO}$ aqueous solution $(\mathrm{O}+\mathrm{W}), \mathrm{W} / \mathrm{O}, \mathrm{O} / \mathrm{W}$, and $\mathrm{BME}$-based solutions under UV light, and a schematic illustration of the suggested dispersion state under each condition. The probe concentration is $1 \mu \mathrm{M}$.

Fig. 5. Results of FCS measurement. Normalized CCFs of (a) $100 \mathrm{nM}$ VE-ATTO and (b) $100 \mathrm{nM}$ VE-BODIPY dispersed in each solution.

Fig. 6. Diffusion times $\tau_{1}$ of VE-ATTO and VE-BODIPY dispersed in each solution. Error bars show the standard deviations $(n=3)$. 


$$
G(\tau)=\frac{1}{N}\left[F_{1}\left(1+\frac{\tau}{\tau_{1}}\right)^{-1}\left(1+\frac{1}{s^{2}} \frac{\tau}{\tau_{1}}\right)^{-\frac{1}{2}}+\left(1-F_{1}\right)\left(1+\frac{\tau}{\tau_{2}}\right)^{-1}\left(1+\frac{1}{s^{2}} \frac{\tau}{\tau_{2}}\right)^{-\frac{1}{2}}\right]+1
$$

Eq. 1 
(a)

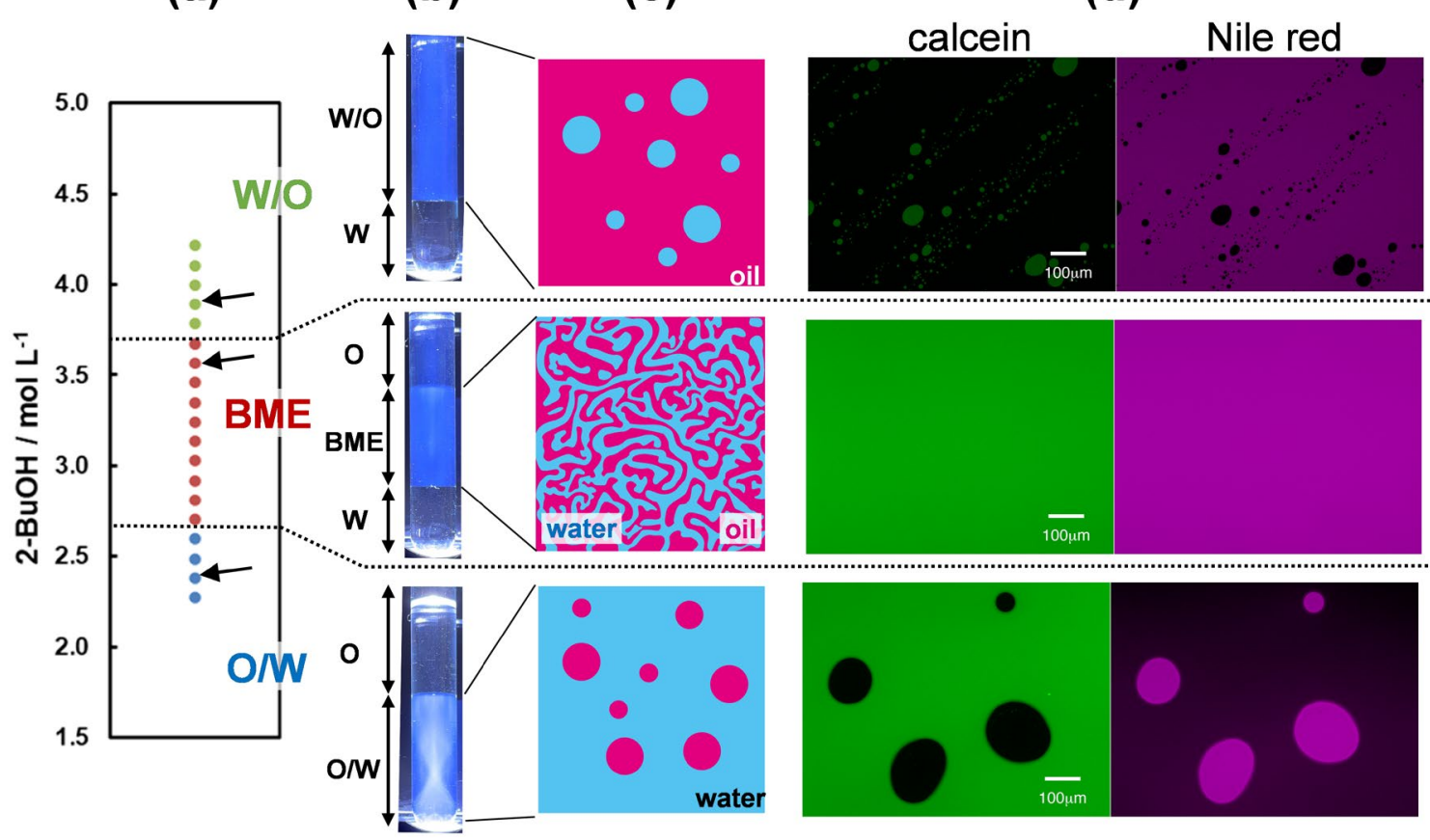

Fig. 1 
(a) VE-ATTO

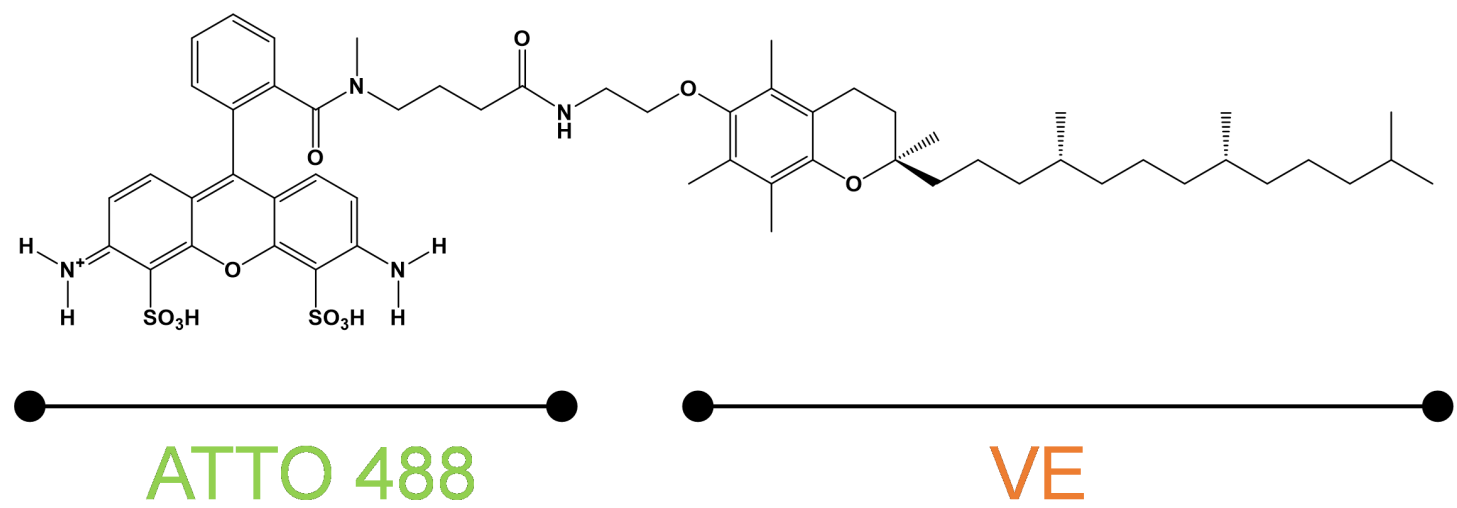

\section{(b) VE-BODIPY}

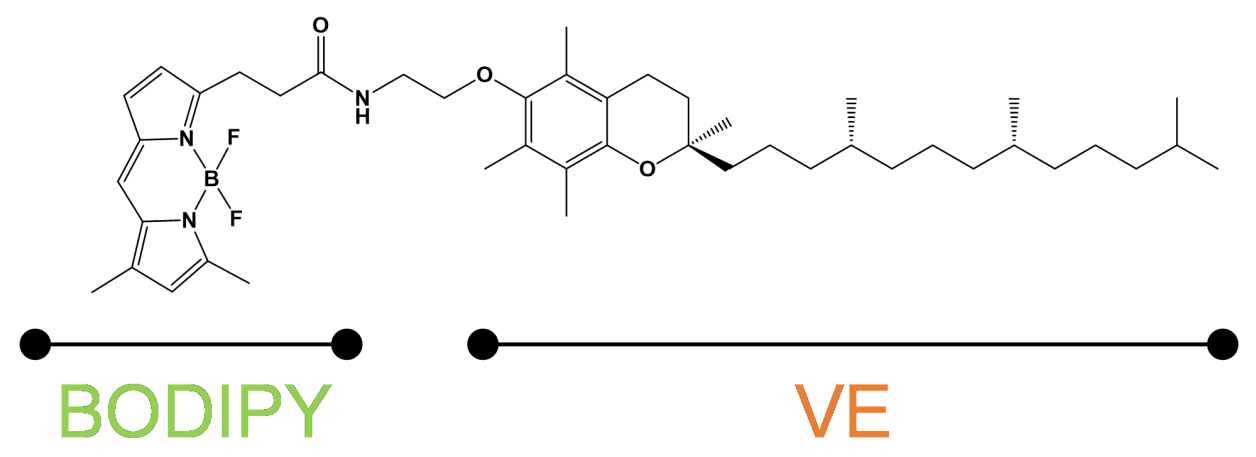

Fig. 2 


\section{(a) VE-ATTO \\ (b) VE-BODIPY}
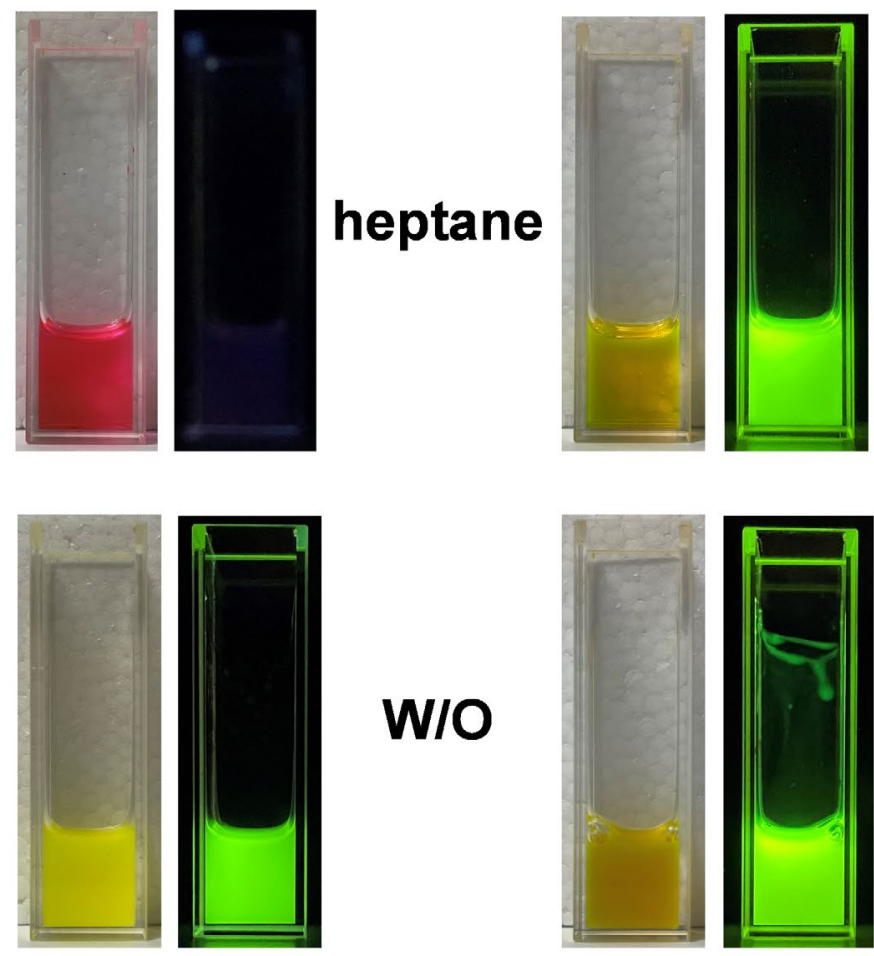

WIO
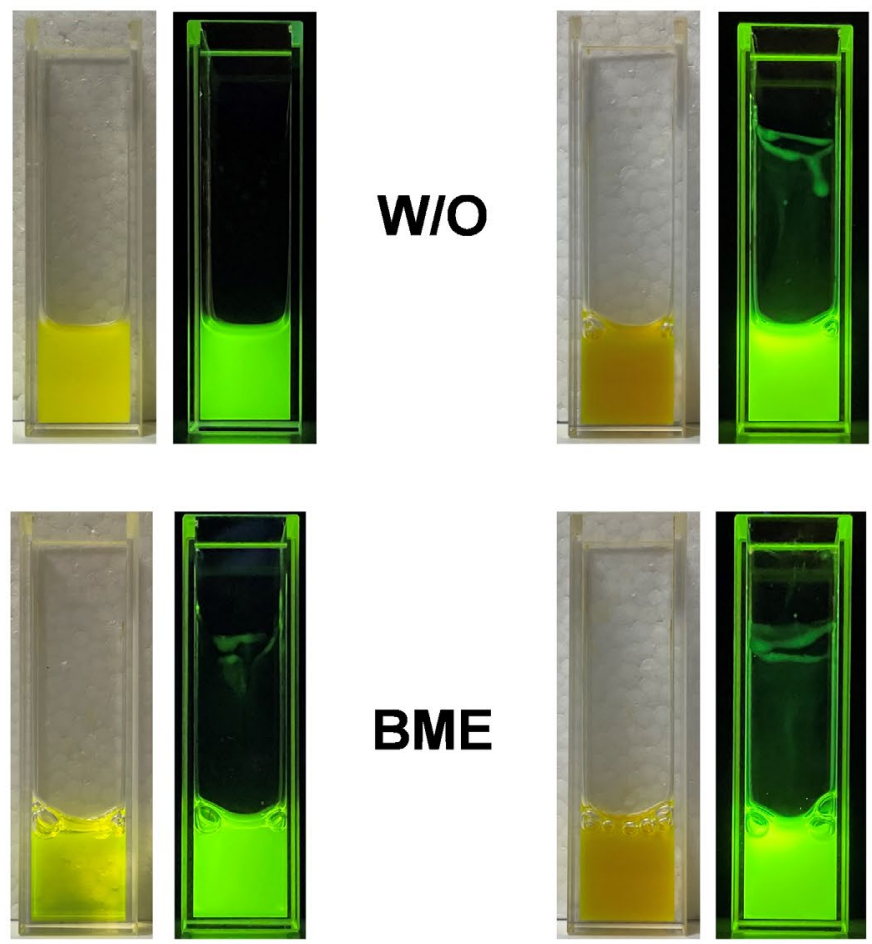

BME
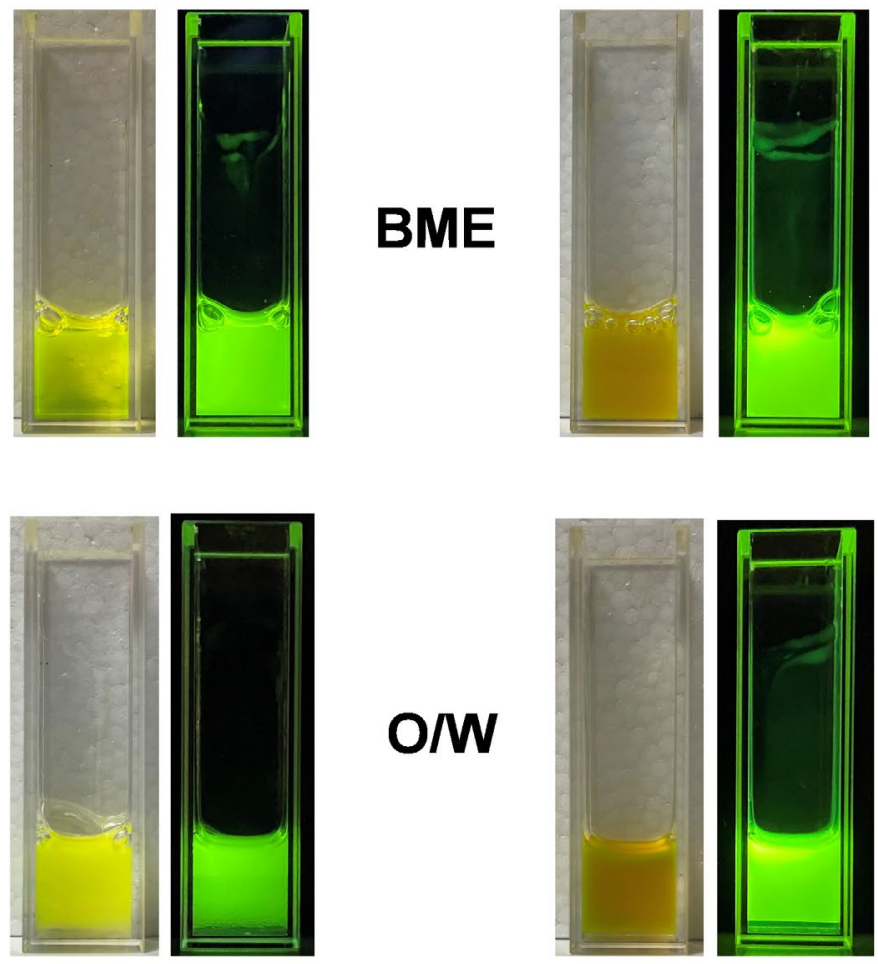

Fig. 3 
(a) VE-ATTO

(b) VE-BODIPY
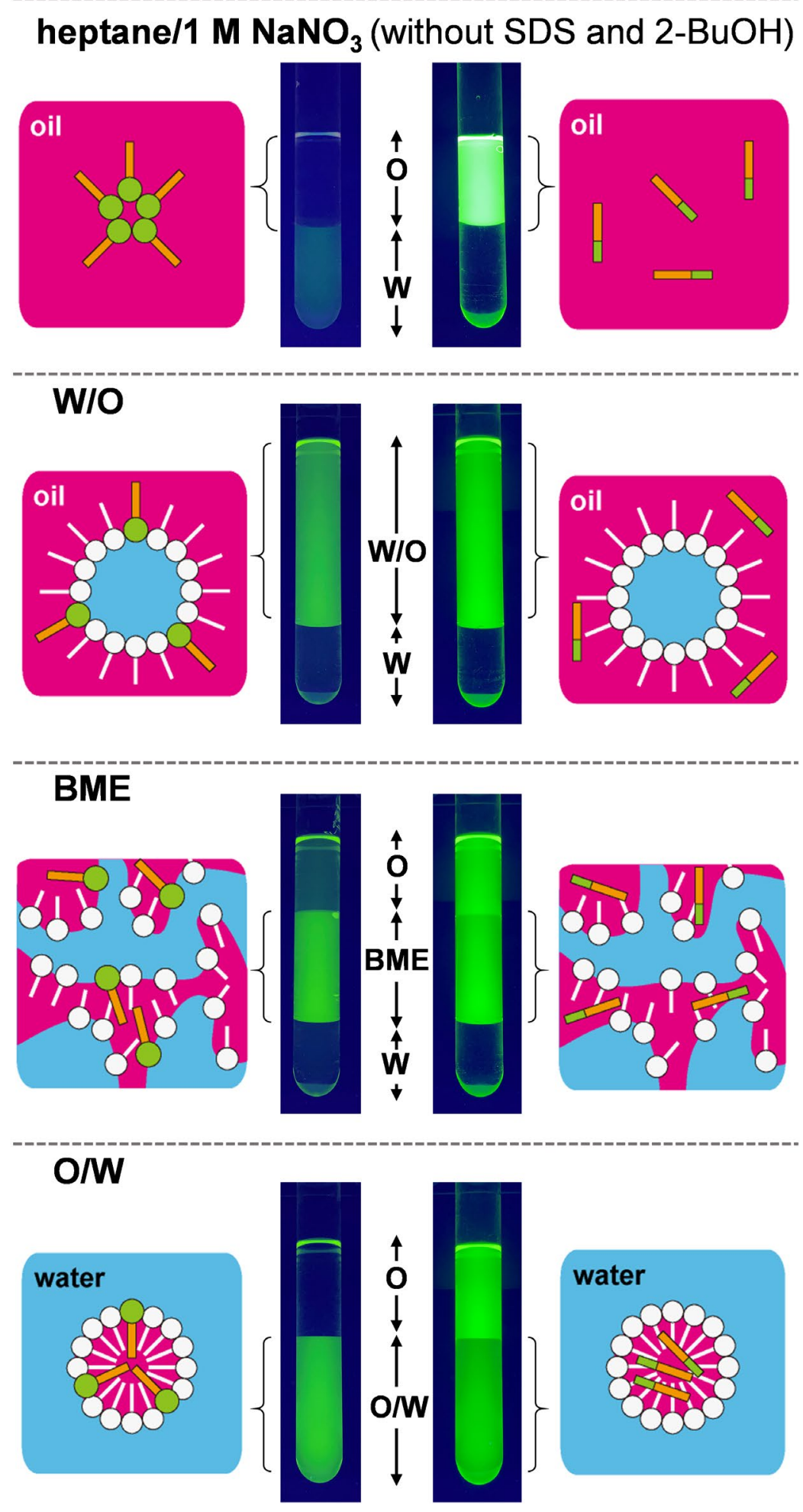

Fig. 4 

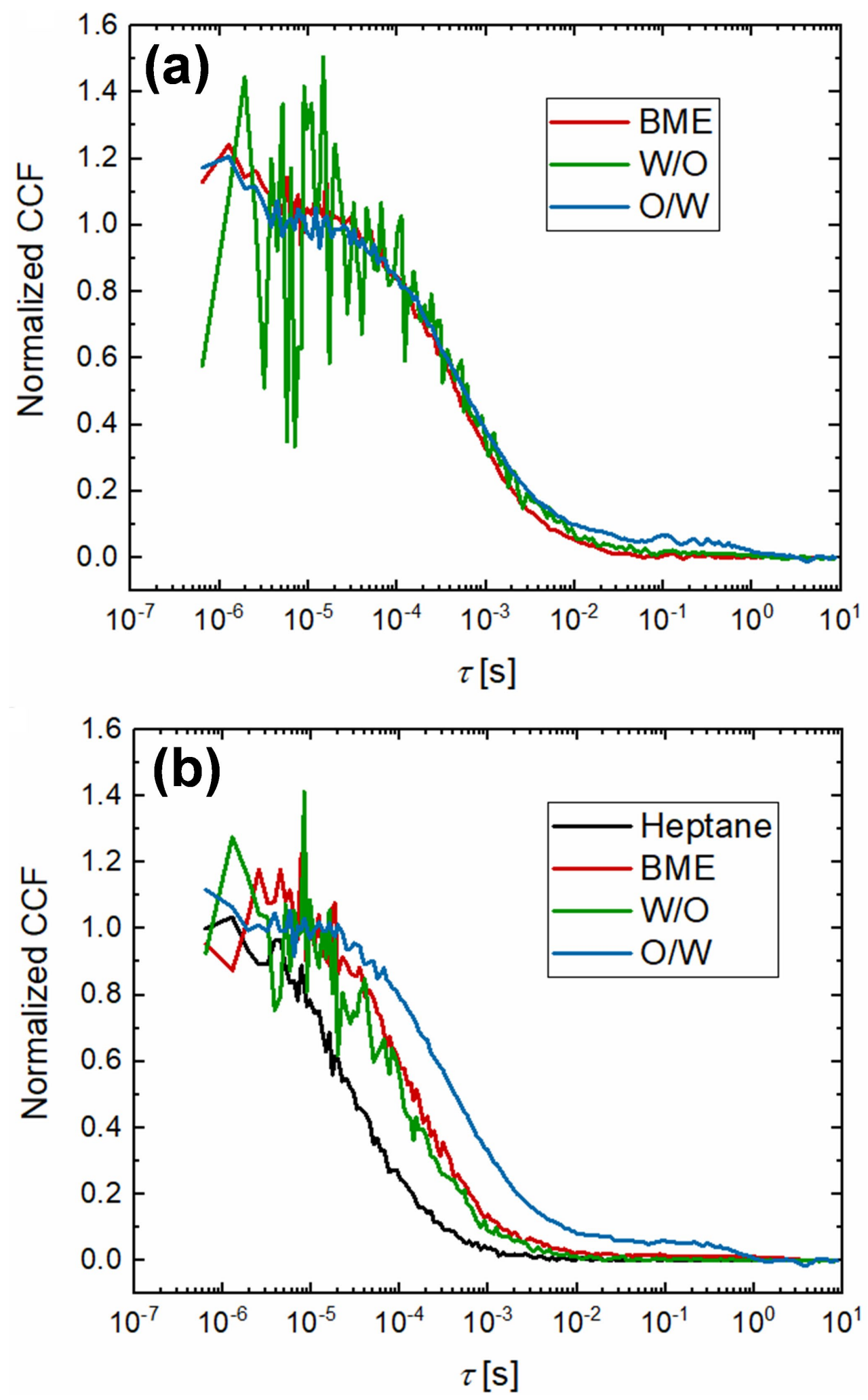

Fig. 5 


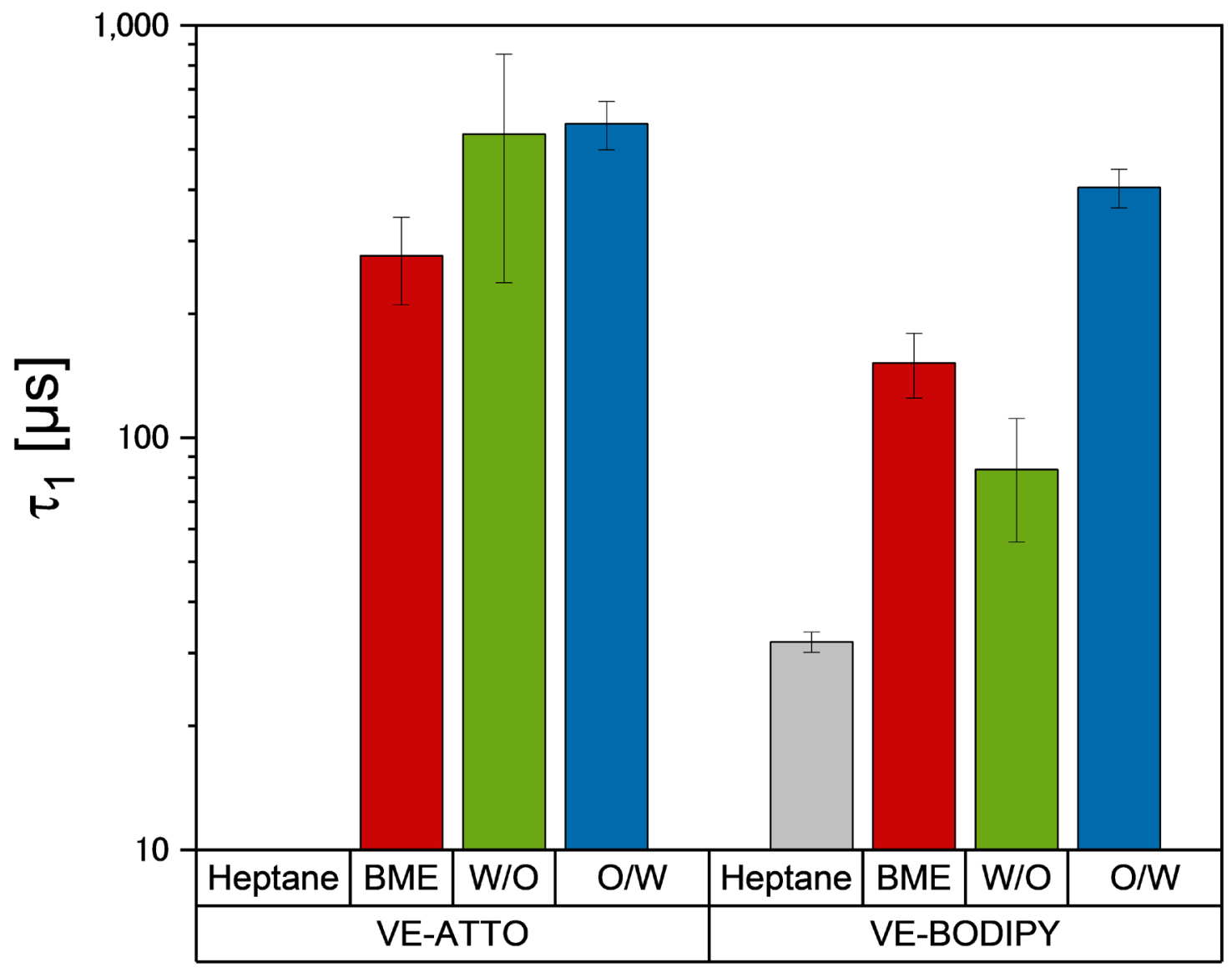

Fig. 6 


\section{Graphical Index}

\section{Lipophilic Probe Behavior in Microemulsions}

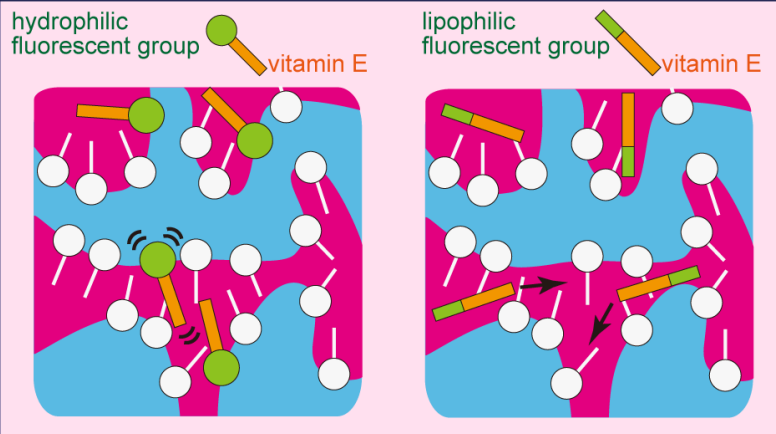

